Proceeding Series of the Brazilian Society of Computational and Applied Mathematics

\title{
Sobre o cálculo dos pontos e dos pesos para a quadratura de Gauss-Gegenbauer quando $0<\lambda<1$
}

\section{Lourenço de Lima Peixoto ${ }^{1}$}

Departamento de Matemática, UFMG, Belo Horizonte, MG,

Formação Geral, IFMG, Congonhas, MG

Resumo. Dois métodos são comparados para o cálculo dos pontos e dos pesos para a quadratura de Gauss-Gegenbauer quando $0<\lambda<1$ : o método do autossistema e o método de Newton-Raphson. A exatidão e o tempo de execução de ambos os métodos são comparados. O método de Newton-Raphson com uma dada aproximação inicial possui convergência garantida e são obtidas desigualdades para os zeros dos polinômios de Gegenbauer.

Palavras-chave. Gauss-Gegenbauer, Autossistema, Newton-Raphson

\section{Introdução}

A quadratura de Gauss-Gegenbauer aproxima integrais do tipo $\int_{-1}^{1}\left(1-x^{2}\right)^{\lambda-\frac{1}{2}} f(x) d x$, $\lambda>-\frac{1}{2}, \lambda \neq 0$. Os $n$ pontos de tal quadratura são os zeros do polinômio de Gegenbauer $P_{n}^{(\lambda)}$ de grau $n$. O método do autossistema consiste em calcular os pontos como sendo os autovalores da matriz de Jacobi cujos autovetores podem ser utilizados para o cálculo dos respectivos pesos. Alternativamente o método de Newton-Raphson (NR) pode calcular os zeros do polinômio $P_{n}^{(\lambda)}$ e os pesos podem ser obtidos por fórmulas conhecidas. Nosso objetivo é comparar a exatidão e o tempo de execução de ambos os métodos quando $0<\lambda<1$, pois a exatidão destes métodos interfere diretamente na aproximação da integral.

\section{Metodologia}

Os pontos e pesos para o autossistema são calculados com a sub-rotina SGAUSQ na linguagem FORTRAN disponível em netlib.org/go/sgausq.f a qual utiliza o algoritmo de [1] que é o mais indicado para o autossistema. Tal sub-rotina foi criada especificamente para este fim. (Uma rotina em MATLAB que implementa o algoritmo de [1] com a função eig é menos eficiente). Elaboramos uma sub-rotina em FORTRAN que implementa o algoritmo do método de NR. Ambas sub-rotinas foram executadas com precisão dupla para diversos valores de $n$ e $\lambda \in(0,1)$. Para comparar a exatidão utilizamos a seguinte medição

\footnotetext{
${ }^{1}$ lourencolp@ufmg.br, lourenco.peixoto@ifmg.edu.br
} 
para o erro $\varepsilon\{V\}$ de um vetor $V=\left(v_{1}, \ldots, v_{n}\right)$, a saber $\varepsilon\{V\}=\max _{1 \leq k \leq n}\left|\frac{v_{k}-v_{k}^{\text {exato }}}{v_{k}^{\text {exato }}}\right|$, $v_{k}^{\text {exato }} \neq 0$. Calculamos $\varepsilon\{X\}$ e $\varepsilon\{W\}$ onde os elementos dos vetores $X$ e $W$ são os pontos e os pesos para um dado $n$. O valor exato $v_{k}^{\text {exato }}$ é considerado aquele fornecido pela subrotina SGAUSQ com precisão estendida. A Tabela 1 exibe os resultados dos erros cometidos pelos dois métodos para alguns valores de $n$ quando $\lambda=0,8$ além do tempo gasto por eles em segundos. Resultados semelhantes foram obtidos com outros valores de $\lambda \in(0,1)$.

Tabela 1: Erros cometidos e tempo em segundos de ambos os métodos quando $\lambda=0,8$.

\begin{tabular}{|c|c|c|c|c|c|c|}
\hline$n$ & $\varepsilon\left\{X_{\text {aut }}\right\}$ & $\varepsilon\left\{X_{N R}\right\}$ & $\varepsilon\left\{W_{\text {aut }}\right\}$ & $\varepsilon\left\{W_{N R}\right\}$ & $\mathrm{t}(\mathrm{s})_{\text {aut }}$ & $\mathrm{t}(\mathrm{s})_{N R}$ \\
\hline 144 & $5,069 \cdot 10^{-14}$ & $4,916 \cdot 10^{-16}$ & $1,072 \cdot 10^{-12}$ & $2,011 \cdot 10^{-13}$ & 0,00284 & 0,00060 \\
987 & $5,564 \cdot 10^{-14}$ & $8,144 \cdot 10^{-16}$ & $6,942 \cdot 10^{-11}$ & $1,428 \cdot 10^{-11}$ & 0,05133 & 0,00855 \\
10946 & $3,723 \cdot 10^{-12}$ & $4,156 \cdot 10^{-15}$ & $8,619 \cdot 10^{-9}$ & $4,927 \cdot 10^{-10}$ & 5,53066 & 0,73391 \\
\hline
\end{tabular}

\section{Conclusões}

Concluimos que o método de NR fornece maior exatidão tanto para os pontos quanto para os pesos, além de ser mais rápido. Em $[3,5]$ encontram-se comparações entre o método do autossistema e outros métodos quanto à exatidão deles quando $\lambda=1 / 2$. Tais autores concluem que o método do autossistema definitivamente não é o mais preciso quando $\lambda=1 / 2$. Nós comparamos o método de NR com o método do autossistema no intervalo $0<\lambda<1$ obtendo semelhante conclusão. Entretanto, o método de NR requer uma boa aproximação inicial para os zeros do polinômio de Gegenbauer. Demonstramos que, com uma fórmula indicada por nós para a aproximação inicial, o método de NR sempre converge. Consequentemente estabelecemos uma desigualdade inferior para cada um dos zeros positivos do polinômio de Gegenbauer quando $2<\lambda<3$. Tal desiguldade é forte e se mostra melhor do que a desigualdade apresentada em [2] para o menor zero positivo e também melhor do que uma desigualdade dada em [4] para o maior zero positivo.

\section{Referências}

[1] G. H. Golub and J. H. Welsch, Calculation of Gauss Quadrature Rules, Mathematics of Computation, vol. 23, 221-230+s1-s10, (1969).

[2] A. Laforgia, Sugli Zeri delle Funzioni di Bessel, Calcolo, vol. 17, 211-220, (1980).

[3] P. N. Swarztrauber, On Computing the Points and Weights for Gauss-Legendre Quadrature, SIAM Journal on Scientific Computing, vol. 24, 945-954, (2003).

[4] G. Szegö, Orthogonal Polynomials, American Mathematical Society, 4a ed., (1975).

[5] E. Yakimiw, Accurate Computation of Weights in Classical Gauss-Christoffel Quadrature Rules, Journal of Computational Physics, vol. 29, 406-430, (1996). 